\title{
Polymorphism of Vesicles with Multi-Domain Patterns
}

\author{
Erwin Gutlederer, Thomas Gruhn, and \\ Reinhard Lipowsky \\ Theory \& Bio-Systems, MPI of Colloids and Interfaces, \\ 14424 Potsdam, Germany*
}

April 5, 2009

\begin{abstract}
Vesicle membranes with two coexisting fluid phases can exhibit a variety of domain patterns. These patterns and the associated vesicle shapes are studied theoretically by minimization of energy functionals that depend on the membrane composition and on the material parameters of the membrane. The latter parameters are (i) the bending rigidities and (ii) the Gaussian curvature moduli of the two types of membrane domains as well as (iii) the line tension of the domain boundaries. It is shown that the interplay between these different parameters leads to stable multi-domain patterns with more than two domains for a wide range of membrane composition and material parameters. As the membrane composition or the material parameters are varied, the vesicles can undergo transitions between different patterns of membrane domains. For fixed vesicle volume, an additional domain-induced transition is observed, in which the vesicle shape changes even though the domain pattern does not. The different multi-domain patterns and vesicle shapes are summarized in terms of morphology diagrams.
\end{abstract}

\section{Introduction}

From the theoretical point of view, biomimetic and biological membranes with several components represent 2-dimensional liquid mixtures and, thus, should exhibit 2-phase coexistence regions, in which the membranes undergo phase separation and form two types of fluid domains $[1,2]$. Even though this view seems rather plausible, it remained controversial for many years, since there was no direct experimental evidence for intramembrane domains. This has changed recently with the introduction of new optical techniques and fluorescent dyes

\footnotetext{
*http://www.mpikg.mpg.de/th/
} 
$[3,4,5,6,5,8,9]$ which have led to the direct visualization of fluid domains on lipid vesicles.

The multi-component model membranes as studied in $[3,4,5,6,5,8,9,10]$ consisted of a saturated lipid such as sphingomyelin, an unsaturated phospholipid, and cholesterol. At appropriate conditions, the presence of cholesterol leads to a separation of the lipids into two fluid phases, a liquid-disordered phase, which is rich in the low-melting point lipid, and a liquid-ordered phase, enriched with cholesterol and the high-melting point lipid. The same lipid mixtures were proposed to form small domains, so-called 'lipid rafts', in the plasma membrane of cells $[11,12]$. These rafts could be relevant for many biological processes, such as cell-signaling and traffic but direct imaging of these latter domains has been difficult, and both the existence and the size of these domains is still a matter of debate.

The morphology of a vesicle with two coexisting fluid phases depends on the membrane composition and on the material properties of the membrane phases as has been previously shown both by analytical theories $[1,2,13]$ and by computer simulation $[14,15,16]$. The most important material parameters are the bending moduli of the two phases, the difference in the Gaussian moduli, and the line tension of the domain boundaries, the latter being first considered in [1]. All of these material parameters provide a contribution to the total energy of the vesicle membrane. The interplay and competition of the different energetic contributions gives rise to morphological transitions. One example is provided by weakly curved membrane domains that form buds as soon as the domain size is sufficiently large. $[1,17,18]$ This domain-induced budding transition is primarily caused by the line tension of the domain boundary and facilitated by a nonzero spontaneous curvature of the membrane domain.

In experiments, a variety of different domain shapes has been observed. If the membranes were flat, the equilibrium state of the two coexisting phases should correspond to two large domains. However, in some experiments, domain patterns with more than two domains have been found to be relatively stable, see, e.g., [4]. Many of these multi-domain patterns are metastable, but in some cases, these metastable patterns are rather long-lived and, thus, kinetically trapped [19]. So far, the analytically calculated domain patterns have been restricted to vesicles with only two domains, beginning with a circular domain in a nearly planar membrane [1], two domains in vesicles with axial symmetry $[2,13]$, and simple domain shapes in membranes with more complex topology [20]. Thus, at present, one cannot decide whether the experimentally observed domain patterns are long-lived metastable states or if they represent the true equilibrium states of the vesicle.

In this article, we study vesicles with spherical topology and address the stability of multi-domain patterns consisting of more than two domains. We find that such patterns are, in fact, stable for a wide range of membrane compositions and material parameters. We focus on membranes with vanishing spontaneous curvature for which the multi-domain patterns and vesicle shapes depend on three dimensionless material parameters. In addition, as one varies the membrane composition or a material parameter, the multi-domain patterns 
undergo transitions from one pattern to another. These domain pattern transitions are coupled to shape transitions and are conveniently summarized in terms of morphology diagrams.

In the context of lipid monolayers at the air-water interface, stable multidomain patterns have been frequently observed [21], which may reflect effective repulsive interactions between the monolayer domains arising from long-ranged dipole-dipole forces [22]. It is important to emphasize that the multi-domain patterns described here do not arise from such long-ranged domain-domain interactions but from the competition of different elastic energies that depend on several elastic parameters such as the bending rigidities of the two membrane domains and the line tension of the domain boundaries. The latter competition is also responsible for the stabilization of striped domain patterns in membranes adhering to corrugated substrates as observed experimentally in [23, 24, 25] and explained theoretically in [26].

Our article is organized as follows. In Sec. 2, we describe the model system of the two-phase vesicle. Especially, the energy expressions and the material parameters are introduced. We have numerically determined the minimum energy configurations for vesicles with different material parameters and constraints. Results are shown in Sec. 3, where we first discuss unconstrained vesicles which can adapt their optimum volume. Volume changes can be strongly restricted by an osmotic pressure, which is induced by molecules in- and outside the vesicle that cannot penetrate the membrane. Morphologies of vesicles with a fixed volume are presented in Sec. 3.4. One focus lies on configurations with more than two domains, which are shown to become relevant for small line tensions. Morphological diagrams of these vesicles are determined as a function of the area fraction, the bending rigidities, and the Gaussian curvature moduli of the two fluid phases.

\section{Theory for multi-domain vesicles}

\subsection{Elastic continuum theory}

In this subsection, we briefly review the elastic continuum theory for multidomain vesicles as developed in $[1,2,13]$. We consider large (or giant) unilamellar vesicles bounded by membranes that contain domains of two coexisting fluid phases, $(\alpha)$ and $(\beta)$. The linear size of the vesicles is large compared to the membrane thickness, and the membrane curvature is small compared to the inverse membrane thickness. We assume that the phases are well segregated and form domains that have a uniform composition. In addition, we focus on situations in which the domain size is large compared to the thickness of the domain boundaries. This separation of length scales implies that spatial variations of the in-plane membrane structure are restricted to relatively sharp

domain boundaries and that we can describe these boundaries as geometrical lines. $[1,2]$ The state of the membrane is then described by a pattern consisting of $(\alpha)$ and $(\beta)$ domains. In general, the membrane can contain several 
disconnected domains of each phase. We will denote the union of all $(\alpha)$ and $(\beta)$ domains by the two regions $S_{\alpha}$ and $S_{\beta}$, respectively. The surface area $A^{(i)}$ of region $S_{i}$ is independent of membrane curvature and remains constant under shape changes of the membrane. The total surface area $A$ of the vesicle is then given by $A=A^{(\alpha)}+A^{(\beta)}$.

For a vesicle with an arbitrary pattern of $(\alpha)$ and $(\beta)$ domains, the energy can be expressed as $[2,13]$

$$
\mathcal{E}=\mathcal{E}_{\mathrm{be}}^{(\alpha)}+\mathcal{E}_{\mathrm{be}}^{(\beta)}+\lambda^{(\alpha \beta)} \int_{\partial S} d l+\sigma^{(\alpha)} A^{(\alpha)}+\sigma^{(\beta)} A^{(\beta)}+\Delta P V .
$$

with the bending energies

$$
\mathcal{E}_{\mathrm{be}}^{(i)}=\int_{S_{i}} d A\left[2 \kappa^{(i)}\left(M-M_{\mathrm{sp}}^{(i)}\right)^{2}+\kappa_{G}^{(i)} G\right] \quad \text { for } \quad i=\alpha, \beta
$$

which depend on the local mean curvature $M$ and the local Gaussian curvature $G$ of the membrane. The integral $\int_{\partial S} d l$ in $(1)$ extends over all domain boundaries between the two types of domains.

The last three terms in the expression (1) are used to incorporate the constraints on the surface areas $A^{(\alpha)}$ and $A^{(\beta)}$ of the $(\alpha)$ and $(\beta)$ domains and on the vesicle volume $V$. For a given temperature, both the surface areas $A^{(\alpha)}$ and $A^{(\beta)}$ and the volume $V$ attain certain, fixed values which are incorporated in the theory via the Lagrange parameters $\sigma^{(\alpha)}, \sigma^{(\beta)}$, and $\Delta P$. Alternatively, the latter parameter may be viewed as the osmotic pressure difference between the exterior and interior vesicle compartments.

For uniform membranes composed of a single phase, the form of the bending energy as given by (2) has been introduced by Helfrich [27]. This bending energy represents the leading order terms of an expansion in powers of the mean and Gaussian curvatures. The truncation of this expansion after the leading terms is justified as long as the membrane curvature is small compared to the inverse membrane thickness. This separation of length scales applies to the morphology of large vesicles but not to membrane nanostructures such as fusion pores or necks which have a size of the order of $10 \mathrm{~nm}$. If one wanted to use an elastic continuum model for the latter structures, one would have to include higher order terms of the curvature expansion [28] and, thus, introduce additional elastic parameters.

\subsection{Material parameters}

In general, the energy of multi-domain vesicle as given by (1) depends on several material parameters: the bending rigidities $\kappa^{(\alpha)}$ and $\kappa^{(\beta)}$, the spontaneous curvatures $M_{\mathrm{sp}}^{(\alpha)}$ and $M_{\mathrm{sp}}^{(\beta)}$, the Gaussian curvature moduli $\kappa_{G}^{(\alpha)}$ and $\kappa_{G}^{(\beta)}$, and the line tension $\lambda^{(\alpha \beta)}$ of the domain boundaries. The three elastic parameters for uniform membranes were introduced in [27], the line tension of the domain boundaries in [1].

The bending rigidity $\kappa$ is the basic energy scale for the bending energy arising from the local mean curvature $M$ of the membrane. For phospholipid bilayers, 
a typical value for $\kappa$ is around $10^{-19} \mathrm{~J}$, see Table 1 in [29], which is of the order of 10-20 $T_{0}$ at room temperature $T_{0}$ (here and below, we absorb the Boltzmann constant $k_{B}$ into the symbol $T$ and measure the temperature in energy units).

The spontaneous curvature $M_{\mathrm{sp}}$ describes the preferred curvature of the membrane and may originate from any bilayer asymmetry such as different lipid compositions of the two leaflets of the bilayer or different aqueous solutions on the two sides of the membrane. In the following, we will focus on bilayers that have the same lipid composition in both leaflets and take $M_{\mathrm{sp}}^{(\alpha)}=M_{\mathrm{sp}}^{(\beta)}=0$. This also implies that we consider intramembrane domains that extend across both leaflets of the bilayer.

The Gaussian curvature modulus $\kappa_{G}$ is difficult to understand intuitively. If the membrane has uniform composition and vanishing spontaneous curvature, the bending energy (2) of two or more vesicles exceeds the energy of one vesicle if $\kappa_{G} / \kappa>-2$. Furthermore, if such a membrane forms a periodic minimal surface with mean curvature $M=0$, the corresponding bending energy (2) is negative if $\kappa_{G}>0$. This indicates that a single vesicle is stable for $-2<\kappa_{G} / \kappa<0$. [30] For monolayers, the stronger stability criterion $-1<\kappa_{G} / \kappa<0$ has been proposed in [31].

For a multi-domain membrane, the Gaussian curvature leads to a contribution along the domain boundaries which is proportional to the difference $\kappa_{G}^{(\alpha)}-\kappa_{G}^{(\beta)}$ of the Gaussian curvature moduli in the two membrane phases $(\alpha)$ and $(\beta)$ as first pointed out in [2]. Two groups have compared the experimentally observed shapes of two-domain vesicles with those calculated from the theory in [2, 13] and deduced the values $\kappa_{G}^{(\alpha)}-\kappa_{G}^{(\beta)} \simeq 3.9 \times 10^{-19} \mathrm{~J}[6]$ and $\kappa_{G}^{(\alpha)}-\kappa_{G}^{(\beta)} \simeq 3 \times 10^{-19} \mathrm{~J}$ [10] for the difference of the Gaussian curvature moduli.

The third term in (1) represents the line energy of the domain boundaries $\partial S$ as introduced in [1] for domains in bilayer membranes. This energy is proportional to the total length of these boundaries, and the energy per unit length defines the line tension $\lambda^{(\alpha, \beta)}$. For lipid monolayers at the air-water interface, domain formation has been observed for many years, see, e.g., [21], and the line tension has been estimated in various ways, see, e.g., [32]. For lipid bilayers, one must distinguish bilayer domains from monolayer domains in the bilayer. The boundary of a bilayer domain represents a cut through the whole bilayer, which can be viewed as a narrow interfacial segment. The line tension is then obtained by integrating the corresponding interfacial free energy over the width of this interface which is equal to the thickness of the bilayer membrane at the domain boundary. [1] In general, the thickness of the two membrane domains may be different as observed experimentally for the liquid-ordered and liquid-disordered phases [33]. These thicknesses do not directly enter the elastic membrane model as given by (1) but are taken into account via their influence on the bending rigidities, the Gaussian curvature moduli, and the line tension.

The presence of a line tension $\lambda^{(\alpha, \beta)}$ leads to domain-induced budding as predicted theoretically $[1,2]$ and observed experimentally for three-component membranes $[4,8,6,10]$. For these latter membranes, the line tension is found to 
vary between $10^{-12}$ and $10^{-14} \mathrm{~N}$ for different compositions and temperatures. Since the phase diagram of the three-component membranes contains critical demixing or consolute points, at which the line tension must vanish [1], the relatively small values of the line tension are plausible and indicate that the experimentally chosen compositions are located in the vicinity of these critical points.

\subsection{Bilayer versus monolayer domains}

In principle, domains in a bilayer membrane can form within each leaflet separately. As mentioned, we will focus on bilayer domains that extend across both leaflets of the bilayer. For such bilayer domains, the lipid composition is the same in each leaflet, and the assumption of vanishing spontaneous curvature is reasonable.

Monolayer domains, which extend only across one leaflet of the bilayer, on the other hand, have a different lipid composition in the two leaflets and will, thus, exhibit some spontaneous curvature [34, 1]. Fluid domains with nonzero spontaneous curvature have an increased tendency to form buds [1] and have been extensively studied in Refs. [2, 13, 35, 36, 14, 37, 38, 39], Furthermore, two monolayer domains in the two leaflets can overlap partially. Thus, the corresponding domain pattern involves three different types of domains: monolayer domains in the inner leaflet (in) with spontaneous curvature $M_{\mathrm{sp}}^{(\mathrm{in})}$, monolayer domains in the outer leaflet (ex) with spontaneous curvature $M_{\mathrm{sp}}^{(\mathrm{ex})}$, and overlapping monolayer domains, say (ov), which are equivalent to bilayer domains with vanishing spontaneous curvature $M_{\mathrm{sp}}^{(\mathrm{ov})}=0$. Such a domain pattern would involve several types of domain boundaries and will not be discussed here.

\subsection{Dimensionless model parameters}

It is useful to reduce the number of model parameters by choosing the bending rigidity $\kappa^{(\beta)}$ of the $\beta$ domains to be the basic energy scale and the linear size $R_{0} \equiv \sqrt{A /(4 \pi)}$ of the vesicle to be the basic length scale. The vesicle morphology is then characterized by five dimensionless parameters. Three of these parameters depend only on material properties: (i) The ratio of the bending rigidities in the $(\alpha)$ and $(\beta)$ phase as defined by

$$
\epsilon^{\kappa} \equiv \frac{\kappa^{(\alpha)}}{\kappa^{(\beta)}},
$$

(ii) the reduced difference in the Gaussian curvature moduli

$$
\Delta \epsilon^{\kappa_{G}} \equiv \frac{\kappa_{G}^{(\alpha)}-\kappa_{G}^{(\beta)}}{\kappa^{(\beta)}},
$$

and (iii) the reduced line tension

$$
\lambda \equiv \lambda^{(\alpha, \beta)} \frac{R_{0}}{\kappa^{(\beta)}} .
$$


The reduced line tension $\lambda$ is proportional to the physical line tension $\lambda^{(\alpha, \beta)}$, proportional to the vesicle size $R_{0}$, and inversely proportional to the bending rigidity $\kappa^{(\beta)}$ of the $(\beta)$ domains. Therefore, the quantity $\lambda$ is a measure for the relative importance of line energy and bending energy: For a given vesicle size $R_{0}$, the reduced line tension $\lambda$ increases if the physical line tension $\lambda^{(\alpha, \beta)}$ increases or if the bending rigidity $\kappa^{(\beta)}$ decreases. Note that the dimensionless line tension $\lambda$ can vary over several orders of magnitude: First, the physical line tension can be as large as $10^{-11} \mathrm{~N}$ and can become arbitrarily small close to a critical demixing point of the multi-component membrane; second, the elastic continuum theory used here can be applied to vesicle sizes in the range $100 \mathrm{~nm}$ $\lesssim R_{0} \lesssim 100 \mu \mathrm{m}$.

In addition to the three material parameters, the system studied here involves two geometric parameters, the fraction

$$
\chi^{(\alpha)} \equiv A^{(\alpha)} /\left(A^{(\alpha)}+A^{(\beta)}\right)=A^{(\alpha)} / A
$$

of the membrane area that is covered by phase $(\alpha)$ and the reduced volume

$$
v \equiv \frac{V}{4 \pi R_{0}^{3} / 3} .
$$

\subsection{Different patterns of membrane domains}

In order to determine the equilibrium state of the multi-domain vesicle, we consider vesicle morphologies with five different domain patterns, see Fig. 1(a) - (e). These patterns differ in the number of separate $(\alpha)$ and $(\beta)$ domains and are labeled by $\mathcal{A}_{B}$ where the large Roman numeral $\mathcal{A}=$ I, II, III corresponds to the number of separate $(\alpha)$ domains whereas the Arabic subscript $B=1,2,3$ represents the number of separate $(\beta)$ domains.

The vesicle morphology $I_{1}$ in Fig. 1 (a) corresponds to a membrane with one $(\alpha)$ domain and one $(\beta)$ domain. These two-domain vesicles are axi-symmetric and have been previously studied in $[2,13]$. The other multi-domain vesicles as shown in Fig. 1(b) - (e) contain more than two distinct domains and have not been considered before. The morphology $\mathrm{II}_{1}$ in Fig. 1(b) contains two $(\alpha)$ and one $(\beta)$ domain, the morphology $\mathrm{III}_{1}$ in Fig. $1(\mathrm{c})$ three $(\alpha)$ and one $(\beta)$ domain, etc. For morphology $\mathrm{III}_{1}$ and $\mathrm{I}_{3}$, we assume that the three $(\alpha)$ and the three $(\beta)$ domains have the same area. If the material parameters of the $(\alpha)$ phase differ from those of the $(\beta)$ phase, morphology $\mathrm{II}_{1}$ is physically different from morphology $\mathrm{I}_{2} \cdot{ }^{1}$

\footnotetext{
${ }^{1}$ The vesicle energy as given by (1) is invariant when the names $(\alpha)$ and $(\beta)$ are permuted, i.e. when $\chi^{(\alpha)}$ is transformed into $\chi^{(\beta)}=1-\chi^{(\alpha)}$ and the material parameters $\kappa^{(\alpha)}, \kappa_{G}^{(\alpha)}$, and $M_{\mathrm{sp}}^{(\alpha)}$ are exchanged with the material parameters $\kappa^{(\beta)}, \kappa_{G}^{(\beta)}$, and $M_{\mathrm{sp}}^{(\beta)}$. Under such a transformation, morphology $\mathrm{I}_{1}$ remains $\mathrm{I}_{1}$, whereas morphologies $\mathrm{II}_{1}$ and $\mathrm{III}_{1}$ are transformed into $I_{2}$ and $I_{3}$, respectively.
} 


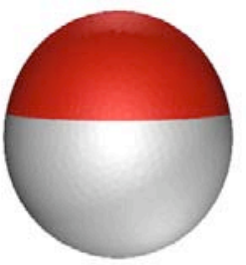

$\mathrm{I}_{1}$

(a)

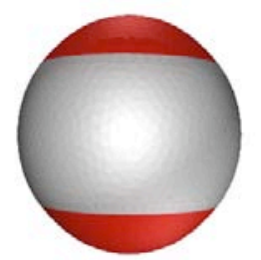

$\mathrm{II}_{1}$

(b)

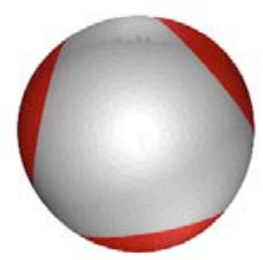

$\mathrm{III}_{1}$

(c)

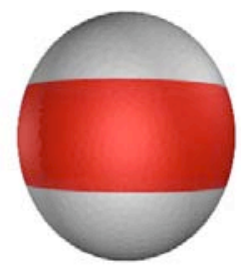

$\mathrm{I}_{2}$

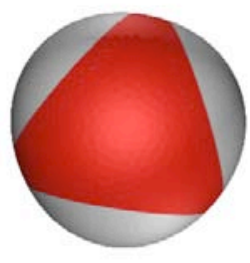

(d)

(e)

$\mathbf{I}_{3}$

Figure 1: Vesicle morphologies with coexisting fluid phases $(\alpha)$ and $(\beta)$ corresponding to the red and white membrane domains, respectively: (a) Domain pattern $\mathrm{I}_{1}$ with one $(\alpha)$ and one $(\beta)$ domain; (b) Pattern $\mathrm{II}_{1}$ with two $(\alpha)$ and one $(\beta)$ domain; (c) Pattern III $_{1}$ with three $(\alpha)$ and one $(\beta)$ domain; (d) Pattern $\mathrm{I}_{2}$ with one $(\alpha)$ and two $(\beta)$ domains; and (e) Pattern $\mathrm{I}_{3}$ with one $(\alpha)$ and three $(\beta)$ domains.

\subsection{Numerical energy minimization}

In this study, we determine and compare the stable and metastable vesicle morphologies as functions of the different system parameters. Vesicle morphologies which are found to have a higher energy are metastable states. The equilibrium morphologies are obtained by direct numerical minimization of a triangulated vesicle surface with the help of the 'Surface Evolver' [40]. Thereby, the surface of the vesicle is implemented as a discretized surface, i.e. a union of triangles, which, mathematically, corresponds to a simplicial complex. The surface evolves towards a minimum energy state via a gradient descent method. During the minimization procedure, the triangulation of the surface is adapted, i.e. refined near regions of high curvature. The estimated numerical accuracy of the obtained energy values is about one percent. We will report results for vesicles with and without volume constraints.

As explained in $[2,13]$, the first variation of the energy functional (1) leads to matching conditions at the domain boundaries that are compatible with any angle between the two tangent planes of the two membrane domains along these boundaries. Thus, one has the freedom to impose an additional boundary con- 
dition that determines this angle. For fluid domains, the most natural condition is provided by the requirement that the membrane shape is smooth across the domain boundary, which implies that the two domains have a common tangent plane. $[2,13]$ This boundary condition was used for all shapes described in the following subsections.

\section{Morphological Diagrams for Multi-Domain Pat- terns}

In this section, vesicle morphologies corresponding to minimal energy are obtained as a function of bending moduli, area fractions, and line tension. In Secs. 3.1-3.3, the vesicle volume is not constrained corresponding to the situation $\Delta P=0$. The influence of the volume constraint is discussed in Sec. 3.4. The results are presented in the form of morphology diagrams, in which the morphologies of lowest energy are shown as a function of the area fraction $\chi^{(\alpha)}$ and of another vesicle parameter. For all cases studied, the morphology $I_{1}$ has the lowest energy for small $\chi^{(\alpha)}$ and for small $1-\chi^{(\alpha)}$, corresponding to a sufficiently small area of $(\alpha)$ and $(\beta)$ phase, respectively.

\subsection{Influence of bending rigidities}

We start with vesicles without volume constraint and first investigate the influence of different bending rigidities $\kappa^{(\alpha)}$ and $\kappa^{(\beta)}$ in the two fluid phases. Without loss of generality, we can assume that $\epsilon^{\kappa}=\kappa^{(\alpha)} / \kappa^{(\beta)} \geq 1$. The results for $\epsilon^{\kappa}<1$ follow directly from those for $\epsilon^{\kappa}>1$ by permuting the names for the $(\alpha)$ and $(\beta)$ phases.

The morphology diagram in Fig. 2(a) shows the multi-domain vesicles with lowest energy as a function of the area fraction $\chi^{(\alpha)}$ and the bending rigidity ratio $\epsilon^{\kappa}$. For vesicles with $\epsilon^{\kappa}$ close to one, i.e., for which both $(\alpha)$ and $(\beta)$ domains have a similar bending rigidity, morphology with the lowest energy is provided by the two-domain pattern $I_{1}$. This is expected because in this state the length of the domain boundary is minimal. As the bending rigidity ratio $\epsilon^{\kappa}$ increases, we find transitions to morphologies with three domains. For $\epsilon^{\kappa}=1.7$, pattern $\mathrm{II}_{1}$ and $\mathrm{I}_{2}$ are favored for $0.2 \lesssim \chi^{(\alpha)} \lesssim 0.5$ and $0.5 \lesssim \chi^{(\alpha)} \lesssim 0.9$, respectively. If $\epsilon^{\kappa}$ is further increased, the $\mathrm{II}_{1}$ region extends towards larger values of $\chi^{(\alpha)}$ and the $\mathrm{I}_{2}$ region shrinks.

In general, the vesicle morphologies with minimal energy are axially symmetric. Typically, the shapes $\mathrm{II}_{1}$ and $\mathrm{I}_{2}$ are found to have an additional up-down (or top-bottom) symmetry, such that the domains located at the two vesicle poles have the same size. Here and below, vesicle morphologies with and without such a symmetry are called nonpolar and polar, respectively. Nonpolar vesicles are favored by bending energy but have a somewhat higher line energy than polar vesicles. Close to the transition between morphology $\mathrm{I}_{1}$ and $\mathrm{II}_{1}$, the energy difference between polar and nonpolar vesicle morphologies of type $\mathrm{II}_{1}$ are smaller than the numerical error for relatively small area fraction $\chi^{(\alpha)}$. Apart 
(a)

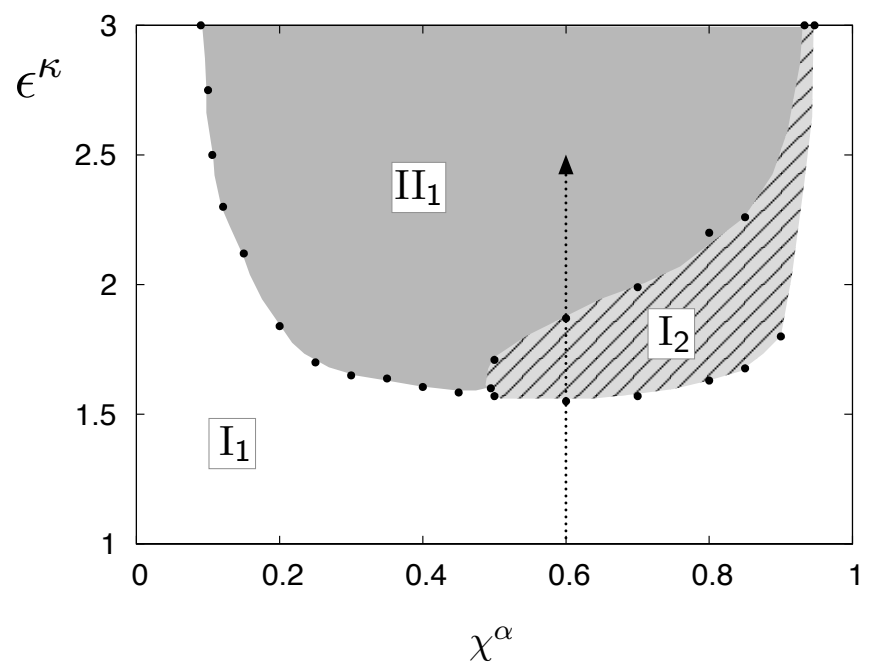

(b)

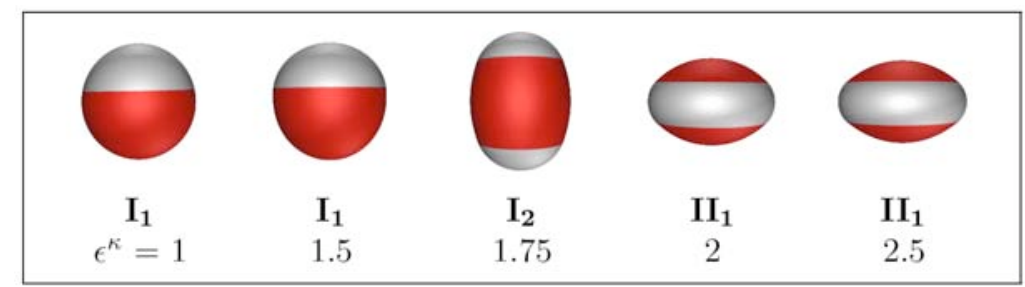

Figure 2: (a) Morphology diagram of multi-domain vesicles without volume constraint as a function of area fraction $\chi^{(\alpha)}$ and bending rigidity ratio $\epsilon^{\kappa}$; and (b) Shapes of minimal energy for $\chi^{(\alpha)}=0.6$ and increasing $\epsilon^{\kappa}$. Both types of domains have the same Gaussian curvature corresponding to $\Delta \epsilon^{\kappa_{G}}=0$, and the domain boundaries are characterized by reduced line tension $\lambda=0.2$. The notation for the multi-domain morphologies is explained in Fig. 1.

from these regions, where our numerical accuracy does not permit a definitive conclusion, our numerical results reveal, that all morphologies with three domains have an up-down symmetry. As a consequence, the transitions shown in the morphology diagram must be discontinuous.

The morphology diagram in Fig. 2(a) is obtained for reduced line tension $\lambda=$ 0.2 . The overall picture does not change qualitatively when the line tension is increased. This is illustrated in the morphology diagram in Fig. 3 corresponding to $\lambda=0.5$. In general, the transition from morphology $\mathrm{I}_{1}$ to morphologies $\mathrm{II}_{1}$ and $\mathrm{I}_{2}$ occurs at larger values of $\epsilon^{\kappa}$ as the line tension increases.

Our results can be understood by inspection of the vesicle configurations in Fig. 2(b) and Fig. 3(b). The $(\alpha)$ domains with the higher bending rigidity avoid conformations with large mean curvature. For small area fraction $\chi^{(\alpha)}$, 
(a)

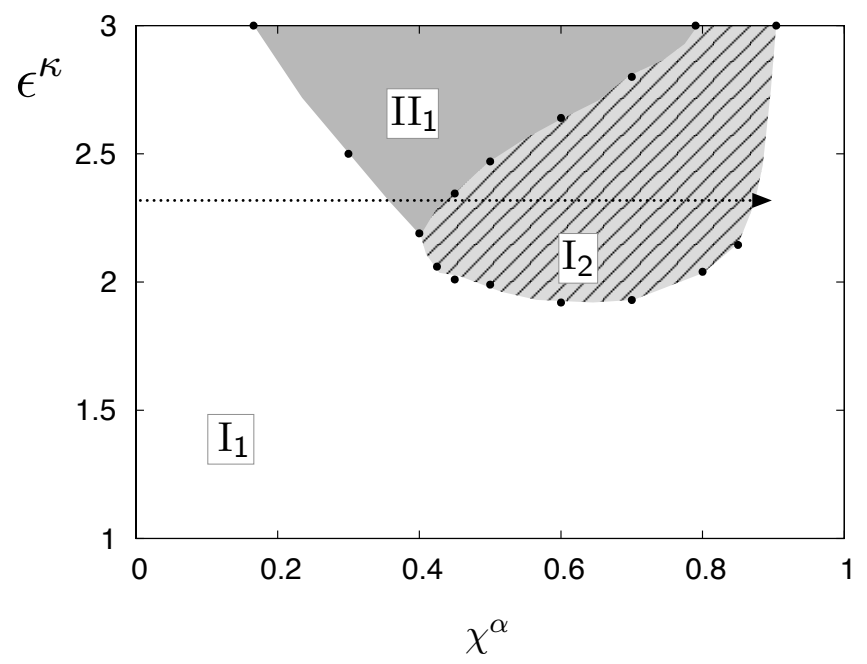

(b)

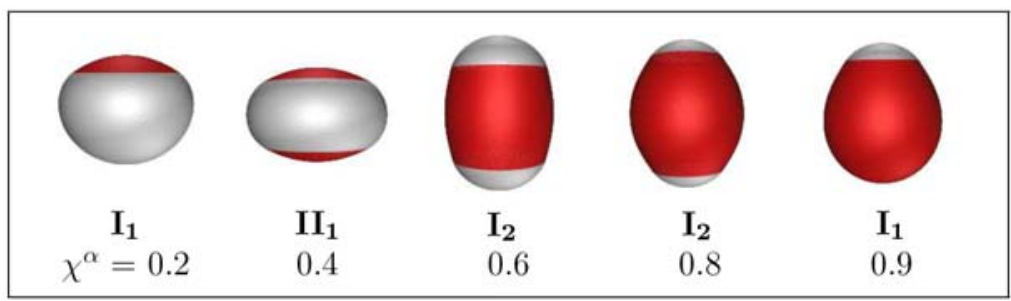

Figure 3: (a) Morphology diagram of multi-domain vesicles without volume constraint as a function of area fraction $\chi^{(\alpha)}$ and bending rigidity ratio $\epsilon^{\kappa}$. The parameters correspond to those in Fig. 2 except for an increased line tension $\lambda=0.5$; and (b) Shapes of minimal energy for $\epsilon^{\kappa}=2.3$ and increasing area fraction $\chi^{(\alpha)}$ as indicated by the arrow in (a). The notation for the morphologies is explained in Fig. 1.

the vesicle morphology $I_{1}$ has the lowest energy because it exhibits the shortest domain boundary. For a small domain size, the stiffer $(\alpha)$ can attain a relatively flat conformation. As the area fraction $\chi^{(\alpha)}$ increases, see Fig. 3(b), morphology $\mathrm{I}_{1}$ becomes less favorable because a single $(\alpha)$ domain at one pole would have to bend more strongly. In order to avoid this increased mean curvature, the vesicle undergoes a transition towards the 3 -domain pattern $\mathrm{II}_{1}$. During this transition, the more rigid $(\alpha)$ domain is divided up into two domains which are bent less and which are located at the poles of the vesicle. The gain in elastic bending energy compensates for the higher line energy arising from the increased length of the domain boundary.

For even larger area fraction $\chi(\alpha)$, morphology $\mathrm{II}_{1}$ is less optimal than morphology $\mathrm{I}_{2}$, and the vesicle undergoes another transition from 3-domain pattern 
$\mathrm{II}_{1}$ to 3 -domain pattern $\mathrm{I}_{2}$. This transition is partly induced by bending energy since morphology $\mathrm{II}_{1}$, with two large domains of the rigid $(\alpha)$ phase, leads to a stronger deformation of the rest of the vesicle than morphology $\mathrm{I}_{2}$ with a single, barrel-like $(\alpha)$ domain. This transition is also favored by line energy, since the domain boundary of the barrel-like morphology $\mathrm{I}_{2}$ starts to be shorter than that of morphology $\mathrm{II}_{1}$ as soon as $\chi(\alpha) \gtrsim 0.5$. This line energy difference also explains why the $\mathrm{I}_{2}$ region increases with increasing line tension $\lambda$ as follows from comparison of Fig. 2(a) for $\lambda=0.2$ with Fig. 3(a) for $\lambda=0.5$.

If one increases the bending rigidity ratio $\epsilon^{\kappa}$ for fixed area fraction, the vesicle can undergo a transition from morphology $\mathrm{I}_{2}$ to morphology $\mathrm{II}_{1}$. An example for such a transition is shown in Fig. 2(b). This transition is governed by the increased bending rigidity of the more rigid $(\alpha)$ phase which overcompensated the increase in line energy as one goes from $\mathrm{I}_{2}$ to $\mathrm{II}_{1}$. Note that the 3-domain pattern $\mathrm{I}_{2}$ with a barrel-like $(\alpha)$ domain has a prolate shape whereas the 3 domain pattern with a belt-like $(\beta)$ domain has an oblate shape.

As mentioned in the introduction, it might be tempting to interpret the multi-domain patterns described here in terms of effective long-ranged interactions between the membrane domains. However, we will now show that such an interpretation is not meaningful. As an example, let us again consider the sequence of shapes as shown in Fig. 2(b). As we increase the bending rigidity ratio $\epsilon^{\kappa}$, we first encounter the transition from morphology $\mathrm{I}_{1}$ with one white $\beta$ domain to morphology $\mathrm{I}_{2}$ with two $\beta$ domains. Now, we might interpret this transition by saying that $\mathrm{I}_{2}$ is characterized by an effective repulsion between the two $\beta$ domains and that this repulsion vanishes along the phase boundary between $\mathrm{I}_{2}$ and $\mathrm{I}_{1}$, compare Fig. 2(a). The location of this phase boundary depends, however, both on the composition variable $\chi_{\alpha}$ and on all the other elastic parameters, which are kept constant in Fig. 2. Therefore, the putative repulsive interaction between the domains would also depend on all of these parameters. In addition, how are we going to interpret the subsequent transition between $\mathrm{I}_{2}$ and $\mathrm{II}_{1}$, which occurs at larger values of $\epsilon^{\kappa}$ ? Should we now say that the effective repulsion between the two $\beta$ domains vanishes again and is simultaneously replaced by an effective repulsion between the two $\alpha$ domains?

Obviously, we do not obtain any additional insight if we try to discuss the multi-domain patterns in Fig. 2 in terms of long-ranged domain-domain interactions. This remark also applies to all other multi-domain patterns as discussed in the following subsections. In fact, our calculation explicitly shows that the multi-domain patterns described here do not arise from long-ranged domaindomain interactions but from the competition of different elastic energies that depend on several elastic parameters such as the bending rigidities of the two membrane domains and the line tension of the domain boundaries. In the next subsection, we will show that these multi-domain patterns are also determined by the Gaussian curvature moduli. 

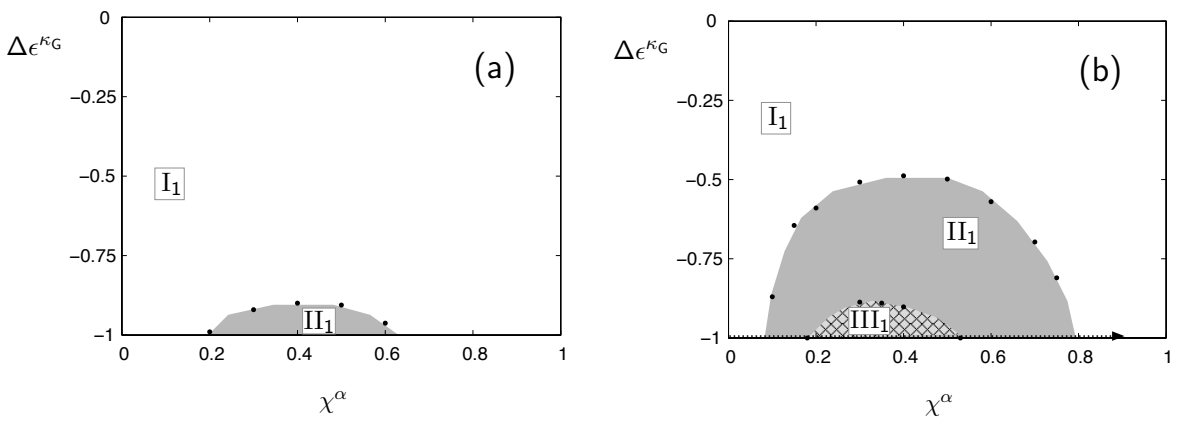

(c)

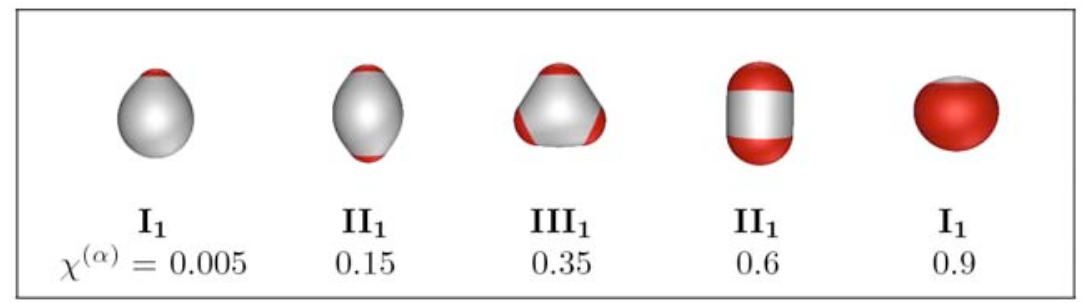

Figure 4: (a,b) Morphology diagrams of multi-domain vesicles without volume constraint as a function of area fraction $\chi^{(\alpha)}$ and reduced difference of the Gaussian curvature moduli, $\Delta \epsilon^{\kappa_{G}}$, as defined in (4). The reduced line tension $\lambda=0.2$ and the bending rigidity ratio has the values $\epsilon^{\kappa}=1$ in (a) and $\epsilon^{\kappa}=0.8$ in (b); and (c) Shapes of minimal energy for $\Delta \epsilon^{\kappa_{G}}=-1, \epsilon^{\kappa}=0.8$, and increasing area fraction $\chi^{(\alpha)}$, as indicated by the arrow along the $\mathrm{x}$-axis in (b). The notation for the morphologies is explained in Fig. 1.

\subsection{Influence of Gaussian curvature moduli}

So far, contributions from the Gaussian curvature moduli of the two phases have been neglected. For a vesicle with a uniform membrane and fixed topology, this is justified because the corresponding bending energy is constant and independent of the size or shape of the vesicle. For a multi-domain vesicle, on the other hand, differences in the Gaussian curvature moduli of the domains give rise to a shape dependent contribution to the bending energy. [2, 13] Using the Gauss-Bonnet theorem for surfaces with boundaries, see, e.g., [41], the bending energy contribution that involves the integral over the Gaussian curvature can be reformulated as an integral over the domain boundaries. This bending energy contribution is proportional to the difference of the Gaussian curvature moduli and to the line integral over the geodesic curvature of the domain boundary. Since this latter integral is dimensionless, it cannot be proportional to the length of the domain boundary and, thus, cannot be viewed as an effective line tension.

For domain-induced budding, the difference in Gaussian curvature moduli 
leads to a displacement of the domain boundary away from the neck. As shown in [13], the domain boundary is shifted towards the domain with the smaller Gaussian curvature modulus and the neck is occupied by the domain with the larger Gaussian curvature modulus. This implies that the local energy density as given by $\kappa_{G}^{(i)} G$ decreases since $G<0$ in the neck region. This conclusion is independent of the sign of the individual Gaussian curvature moduli.

In the following, we investigate how the multi-domain patterns are affected by a difference in Gaussian curvature moduli corresponding to a nonzero value of $\Delta \epsilon^{\kappa_{G}}$ as defined in (4). We first consider a vesicle with $(\alpha)$ and $(\beta)$ domains that have equal bending rigidities corresponding to $\epsilon^{\kappa}=1$, which implies that the two membrane phases are identical apart from their Gaussian curvature moduli. The morphology diagram of such vesicles is shown in Fig. 4(a) as a function of area fraction $\chi^{(\alpha)}$ and reduced Gaussian bending modulus $\Delta \epsilon^{\kappa_{G}}$ for reduced line tension $\lambda=0.2$. More precisely, Fig. 4(a) displays the morphological diagram only for negative values of $\Delta \epsilon^{\kappa_{G}}$ or for $\kappa_{G}^{(\alpha)}<\kappa_{G}^{(\beta)}$, i.e., for the situation in which the Gaussian bending modulus of the $(\alpha)$ phase is smaller than the one of the $(\beta)$ phase. For the case with $\kappa^{(\alpha)}=\kappa^{(\beta)}$ as considered here, it is straightforward to construct the remaining part of the morphology diagram for positive values of $\Delta \epsilon^{\kappa_{G}}$. Indeed, permuting the names of the phases $(\alpha)$ and $(\beta)$ shows that a minimum energy morphology $\mathrm{II}_{1}$ for a vesicle with $\chi^{(\alpha)}=x$ and $\Delta \epsilon^{\kappa_{G}}=y$ implies a minimum energy morphology $\mathrm{I}_{2}$ for a vesicle with $\chi^{(\alpha)}=1-x$ and $\Delta \epsilon^{\kappa_{G}}=-y$. Note that this simple mapping no longer applies if the bending rigidity or any other material property of the phases $(\alpha)$ and $(\beta)$ are different.

For equal bending rigidity, $\epsilon^{\kappa}=1$, and $\kappa_{G}^{(\alpha)}<\kappa_{G}^{(\beta)}$, morphology $\mathrm{I}_{1}$ has the minimum energy for all vesicles with $\left|\Delta \epsilon^{\kappa_{G}}\right| \lesssim 0.9$. For $\left|\Delta \epsilon^{\kappa_{G}}\right| \gtrsim 0.9$ and $0.2 \lesssim$ $\chi^{(\alpha)} \lesssim 0.6$, the diagram shows a region where morphology $\mathrm{II}_{1}$ is energetically favored. In this range, the phase $(\alpha)$, which has the smaller Gaussian curvature modulus, forms two domain caps located at the poles of the vesicle.

The impact of a different bending rigidity ratio, $\epsilon^{\kappa} \neq 1$, can be seen by inspection of Fig. 4(b). We assume that the membrane in phase $(\alpha)$ is softer and easier to bend, i.e. $\epsilon^{\kappa}<1$. Fig. 4(b) displays the morphology diagram for $\epsilon^{\kappa}=0.8$. Since the two membrane phases have different bending moduli, the mapping from negative to positive values of $\Delta \epsilon^{\kappa_{G}}$ as discussed for $\epsilon^{\kappa}=1$ is no longer valid. In fact, we find that the 2-domain pattern $I_{1}$ represents the minimal energy morphology for all positive values of $\Delta \epsilon^{\kappa_{G}}$, even though 3-domain and even 4-domain patterns represent minimal energy morphologies for $\Delta \epsilon^{\kappa_{G}}<0$, see Fig. 4(b). For $\Delta \epsilon^{\kappa_{G}}<-0.5$, the 3-domain pattern $\mathrm{II}_{1}$, which contains two $(\alpha)$ domains, is favored provided the area fraction of the $(\alpha)$ and $(\beta)$ domains are sufficiently large. For $\Delta \epsilon^{\kappa_{G}} \lesssim-0.9$, there is a domain pattern transition towards the 4-domain pattern $\mathrm{III}_{1}$ with three $(\alpha)$ domains and one $\beta$ domain.

Altogether, we find that if a membrane phase has both a smaller Gaussian curvature modulus and a smaller bending rigidity than the other membrane phase, it tends to form several bulge-like domains, characterized both by a large Gaussian curvature and by a large mean curvature. On the other hand, if one 
(a)

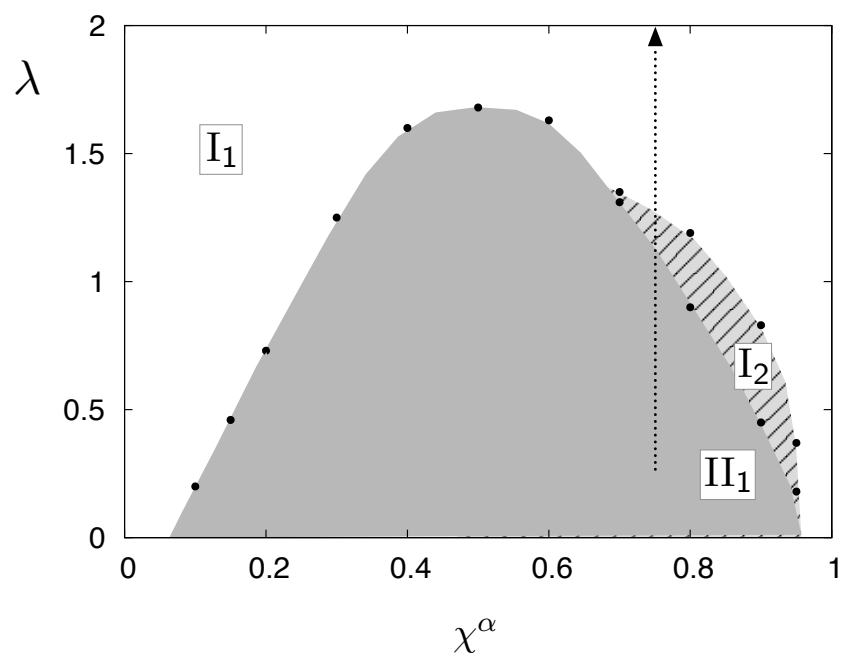

(b)

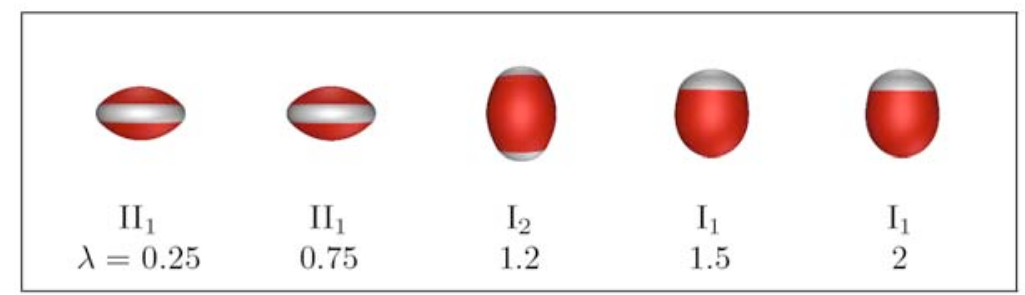

Figure 5: (a) Morphology diagram of multi-domain vesicles without volume constraint as a function of area fraction $\chi^{(\alpha)}$ and reduced line tension $\lambda$ for bending rigidity ratio $\epsilon^{\kappa}=4$ and reduced ratio $\Delta \epsilon^{\kappa_{G}}=-1.5$ of the Gaussian curvature moduli. (b) Shapes of minimal energy for $\chi^{(\alpha)}=0.75$ and increasing $\lambda$, as indicated by the arrow in (a). The notation for the morphologies is explained in Fig. 1.

phase has a smaller Gaussian curvature modulus but a larger bending rigidity, the bending energies compete and the formation of more than two domains is suppressed.

\subsection{Influence of line tension}

Next, we investigate the dependence of the multi-domain patterns on the reduced line tension $\lambda$. In order to do so, we will now focus on the coexistence of liquid-disordered $\left(L_{d}\right)$ and liquid-orderd $\left(L_{o}\right)$ domains as studied experimentally in three-component lipid membranes and identify the more rigid $L_{o}$ domain with the $(\alpha)$ domain and the more flexible $L_{d}$ domain with the $(\beta)$. The material parameters of these two types of domains depend on membrane composition 
and temperature.

As previously mentioned, two experimental groups $[6,10]$ have used the elastic continuum theory developed in $[1,2,13]$ and reviewed in Sect. 2.1 in order to extract values for these material parameters. First, Baumgart et al [6] performed a detailed analysis for a lipid membrane composed of DOPC, egg sphingomyelin, and cholesterol with mole fraction composition 0.135:0.615:0.25 at $30^{\circ}$ C. For this three-component membrane, they estimated the bending rigidity ratio $\epsilon^{\kappa}=\kappa^{\left(L_{o}\right)} / \kappa^{\left(L_{d}\right)} \simeq 5$ and the reduced difference in Gaussian curvature moduli, $\Delta \epsilon^{\kappa_{G}} \simeq-3.6$. Second, Semrau et al [10] have studied lipid membranes composed of DOPC, brain sphingomyelin, and cholesterol with composition 0.3:0.5:02 at $20^{\circ} \mathrm{C}$. For the latter mixture, they obtained estimates for the material parameters which lead to $\epsilon^{\kappa} \simeq 4$ and $\Delta \epsilon^{\kappa_{G}} \simeq-1.5{ }^{2}$

As an example, we have chosen the latter parameter values, $\epsilon^{\kappa}=4$ and $\Delta \epsilon^{\kappa_{G}}=-1.5$, and calculated the corresponding morphology diagram as a function of area fraction $\chi^{(\alpha)}$ and reduced line tension $\lambda$, see Fig. 5. We find that the 2 -domain pattern $I_{1}$ provides the minimal energy morphology for all values of $\chi^{(\alpha)}$ provided the reduced line tension exceeds the threshold value $\lambda^{*} \simeq 1.7$. For $\lambda \lesssim \lambda^{*}$, the 2-domain pattern $\mathrm{I}_{1}$ is found for large and small $\chi^{(\alpha)}$ while the 3 -domain pattern $\mathrm{II}_{1}$ is favored for intermediate values of $\chi^{(\alpha)}$. In addition, the 3 -domain pattern $\mathrm{I}_{2}$ provides the minimal energy morphology in a narrow parameter region along the boundary of the $\mathrm{II}_{1}$ region for relatively large values of $\chi^{(\alpha)}$, see Fig. 5(a).

The reduced line tension $\lambda=\lambda^{(\alpha, \beta)} / R_{0} \kappa^{(\beta)}$ as defined in (5) depends on the physical line tension $\lambda^{(\alpha, \beta)}$, the bending rigidity $\kappa^{(\beta)}$ of the $(\beta)$ or $\left(L_{d}\right)$ domains, and the vesicle size $R_{0}$. For given values of the physical line tension $\lambda^{(\alpha, \beta)}$ and the bending rigidity $\kappa^{(\beta)}$, the threshold value $\lambda^{*}$ for the reduced line tension then implies a threshold value $R_{0}^{*}$ for the vesicle size as given by

$$
R_{0}^{*}=\lambda^{*} \kappa^{(\beta)} / \lambda^{(\alpha, \beta)} .
$$

Large vesicles with $R_{0}>R_{0}^{*}$ exhibit the 2 -domain patterns $\mathrm{I}_{1}$. Small vesicles with $R_{0}>R_{0}^{*}$, on the other hand, can undergo transitions to 3-domain patterns $\mathrm{II}_{1}$ or $\mathrm{I}_{2}$.

Using the value $\lambda^{*}=1.7$ as obtained from the morphological diagram in Fig. 5(a) as well as the values $\lambda^{(\alpha, \beta)} \simeq 1.2 p N$ and $\kappa^{(\beta)} \simeq 2 \cdot 10^{-19} \mathrm{~J}$ as estimated for the three-component membrane studied in [10] at $20^{\circ} \mathrm{C}$, we obtain the threshold value $R_{0}^{*} \simeq 0.28 \mu \mathrm{m}$ for the vesicle size. This threshold value is expected to increase with increasing temperature since the line tension $\lambda^{(\alpha, \beta)}$ should then decrease significantly.

\subsection{Influence of volume constraint}

Finally, we extend our analysis to vesicles which are subject to a volume constraint. For large vesicles, the volume is usually determined by the osmotic

\footnotetext{
${ }^{2}$ All numerical values for $\Delta \kappa_{G}$ as quoted in Table I of Ref. [10] should be multiplied by $(-1)$ (S. Semrau, private communication).
} 
pressure difference arising from the osmotically active molecules inside and outside the vesicle. In the following, we consider the limiting case in which the volume is fixed to a certain value.

(a)

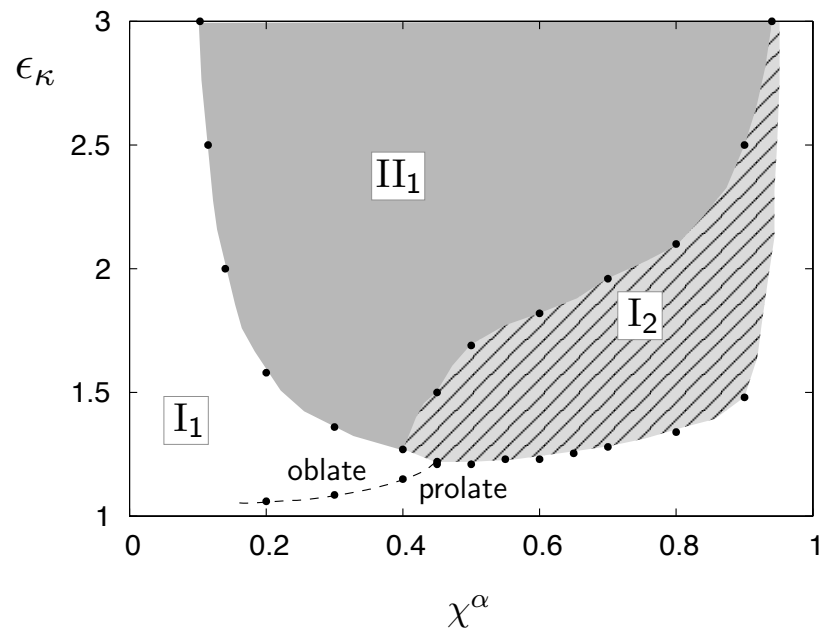

(b)

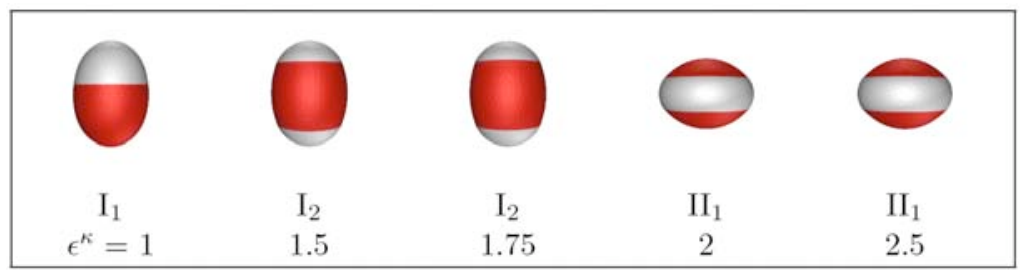

Figure 6: (a) Morphology diagram of multi-domain vesicles with fixed reduced volume $v=0.97$ as a function of area fraction $\chi^{(\alpha)}$ and bending rigidity ratio $\epsilon^{\kappa}$; and (b) Shapes of minimal energy for $\chi^{(\alpha)}=0.6$ and increasing $\epsilon^{\kappa}$. As in Fig. 2, both types of domains have the same Gaussian curvature corresponding to $\Delta \epsilon^{\kappa_{G}}=0$, and the domain boundaries are characterized by reduced line tension $\lambda=0.2$. The notation for the multi-domain morphologies is explained in Fig. 1.

We analyze vesicles with a reduced line tension $\lambda=0.2$ and equal Gaussian bending moduli $\Delta \epsilon^{\kappa_{G}}=0$ for reduced volume $v=0.97$, see Fig. 6 . The same parameter values have been studied in Sec. 3.1 without volume constraint, see Fig. 2. Comparison of Fig. 2(a) and Fig. 6(a) shows that the volume constraint has a relatively small effect on the morphology diagram. For fixed volume, morphologies with more than two domains appear for slightly smaller values of the bending rigidity ratio $\epsilon^{\kappa}$. This shift can be understood by comparing the vesicle shapes in Fig. 2(b) and Fig. 6(b). Without volume constraint, the mutidomain pattern $\mathrm{I}_{1}$ prefers an almost spherical shape with $v \simeq 0.99$ whereas the 
patterns $\mathrm{I}_{2}$ and $\mathrm{II}_{1}$ lead to prolate and oblate shapes and, thus, have a smaller reduced volume $v$.

\subsubsection{Domain-induced transitions between prolates and oblates}

The volume constraint leads to additional morphological transitions of multidomain vesicles. As a simple example, we will demonstrate this additional feature for the $I_{1}$ pattern, for which we observe a domain-induced transition between prolate and oblate shapes.

Let us first consider axisymmetric prolate and oblate shapes with fixed reduced volume $v=0.97$ as denoted by $\mathrm{I}_{1 \text {,pro }}$ and $\mathrm{I}_{1, \text { obl }}$ in Fig. 7(a). The minimal energies of these two shapes are shown in Fig. 7(b) as a function of bending rigidity ratio $\epsilon^{\kappa}$ for area fraction $\chi^{(\alpha)}=0.3$. The other parameters have the same values as in Fig. 2 and Fig. 6. For $\epsilon^{\kappa}<\epsilon_{*}^{\kappa} \simeq 1.08$ and $\epsilon^{\kappa}>\epsilon_{*}^{\kappa}$, the prolate $\mathrm{I}_{1, \text { pro }}$ and the oblate $\mathrm{I}_{1, \text { obl }}$ have minimal energy, respectively.

For the reduced volume $v=0.97$ considered here, a uniform vesicle attains a prolate shape. Therefore, for a two-domain vesicle with $\epsilon^{\kappa}=1$ and relatively small line tension, the shape of minimal energy is provided by the axisymmetric prolate $\mathrm{I}_{1, \text { pro }}$ with a 'cap domain' on its pole. This domain configuration is characterized by a relatively short domain boundary. As the bending rigidity ratio $\epsilon^{\kappa}$ increases, i.e., as the minority phase $(\alpha)$ in Fig. 7(a) becomes more rigid, the vesicle undergoes a transition to an oblate morphology $\mathrm{I}_{1, \mathrm{obl}}$. The transition occurs for area fractions $\chi^{(\alpha)}<0.5$ and bending rigidities slightly above $\epsilon^{\kappa}=1$ as indicated by the dashed transition line in Fig. 6(a).

Far from the prolate-oblate transition line, axisymmetric shapes are energetically favored, and the $(\alpha)$ domain is located at one pole of the vesicles. For $\chi^{(\alpha)}=0.3$, the minimal energy of the vesicle is then given by the lower branches in Fig. 7(b), i.e., by the energy of the prolate shape $\mathrm{I}_{1 \text {,pro }}$ for $\epsilon^{\kappa}<\epsilon_{*}^{\kappa}-\delta=1.08-\delta$ and by the energy of the oblate shape $\mathrm{I}_{1, \mathrm{obl}}$ for $\epsilon^{\kappa}>\epsilon_{*}^{\kappa}+\delta^{\prime}=1.08+\delta^{\prime}$. The two parameters $\delta \geq 0$ and $\delta^{\prime} \geq 0$ indicate that, close to the transition point, the shape of minimal energy may be non-axisymmetric: one example is provided by the shape $I_{1, \text { non }}$ as shown in Fig. $7(a)$. The energy difference between axiand non-axisymmetric shapes is, however, quite small and was below the numerical accuracy of our minimization calculations. Likewise, for small values of

$\chi^{(\alpha)}$, the energy difference between the axisymmetric prolates $\mathrm{I}_{1 \text {,pro }}$ and oblates $\mathrm{I}_{1, \mathrm{obl}}$ becomes so small that we could no longer calculate the transition line numerically. Thus, the precise location of the domain-induced transition between prolates and oblates and the order of this transition remains to be determined.

\section{Conclusions}

We have studied the minimal energy configurations of vesicles bounded by membranes that contain two coexisting fluid phases. The shapes and domain patterns of these vesicles are governed by the interplay between line energy and bending energies. For vanishing spontaneous curvatures as studied here, the 
(a)

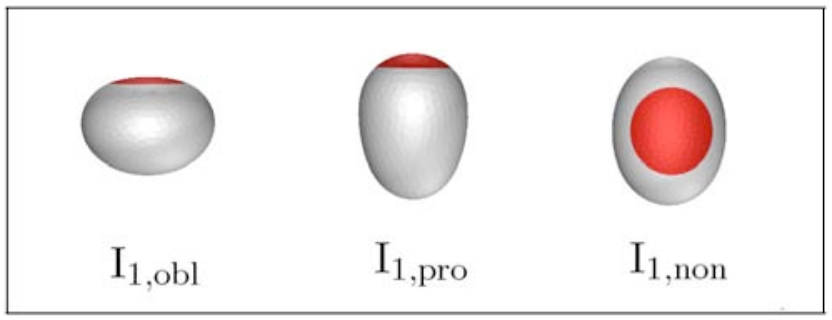

(b)

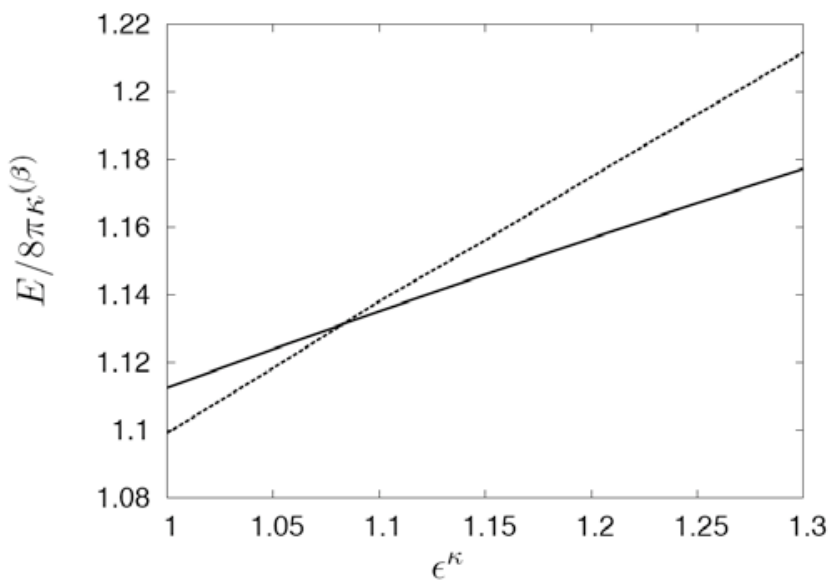

Figure 7: (a) Different shapes for the $I_{1}$ pattern with one $(\alpha)$ and one $(\beta)$ domain: axi-symmetric oblate $\mathrm{I}_{1, \mathrm{obl}}$, axi-symmetric prolate $\mathrm{I}_{1 \text {,pro }}$, and nonaxisymmetric pattern $\mathrm{I}_{1, \text { non }}$; and (b) Energy $\mathcal{E}$ of oblate morphology $\mathrm{I}_{1, \text { obl }}$ (full line) and prolate morphology $\mathrm{I}_{1, \text { pro }}$ (broken line) as a function of bending rigidity ratio $\epsilon^{\kappa}$. The two two types of membrane domains have the same Gaussian curvature modulus; the reduced line tension has the value $\lambda=0.2$, the area fraction $\chi^{\alpha}=0.3$, and the reduced volume $v=0.97$.

shapes and domain patterns depend on three dimensionless material parameters, namely the bending rigidity ratio $\epsilon^{\kappa}$, the reduced difference $\Delta \epsilon^{\kappa_{G}}$ of the Gaussian curvature moduli, and the reduced line tension $\lambda$, as defined in (3) (5) and two geometric parameters, namely the area fraction $\chi^{\alpha}$ and the reduced volume $v$, as defined in (6) and (7).

We find that vesicles with more than two intramembrane domains can have minimal energy and represent the equilibrium morphology for a wide range of parameters, see the different morphological diagrams in Fig. 2(a) - 6(a). These morphological diagrams exhibit domain pattern transitions that can be induced by a variation of line tension, ratio of bending rigidities, or difference in Gaussian curvature moduli. Minimal energy shapes with more than two domains are especially favored by relatively low line tension and intermediate area fractions $\chi^{(\alpha)}$ close to $1 / 2$. These multi-domain shapes are dominant if 
the bending ratio $\epsilon^{\kappa}$ is sufficiently large and the reduced difference $\Delta \epsilon^{\kappa_{G}}$ of the Gaussian curvature moduli is sufficiently negative. For $\epsilon^{\kappa}=0.8, \Delta \epsilon^{\kappa_{G}} \simeq-1$, and $\lambda=0.2$, the pattern $\mathrm{III}_{1}$ consisting of three $(\alpha)$ and one $(\beta)$ domain has been found to be the minimal energy shape for a certain intermediate range of area fractions, see Fig. 4(b) and (c). Our estimates at the end of section 3.3 indicate that these transitions between different multi-domain patterns on vesicles should be accessible to experiment.

In order to eliminate one parameter, we first studied vesicles without a volume constraint; the corresponding morphology diagrams are displayed in Fig. 2(a) - 5(a). However, we have also shown that a volume constraint does not change the qualitative features of the morphological diagrams as can be concluded by comparison of Fig. 6(a) with Fig. 2(a). In fact, the volume constraint was found to lead to additional morphological transitions from prolate to oblate shapes, see Fig. 6(a) and Fig. 7(a).

The multi-domain patterns considered here arise from the competition between different elastic energies of the vesicle membrane as desribed by the energy functional (1). On the one hand, the membrane can lower its elastic energy by decreasing the number of membrane domains since it then reduces the line energy of the domain boundaries. On the other hand, the membrane may also lower its elastic energy by increasing the number of membrane domains if the more flexible and the more rigid domains can be accommodated in the more strongly and the more weakly curved membrane regions, respectively. The same type of competition is also responsible for the stabilization of striped domain patterns in membranes adhering to corrugated substrates as observed experimentally in $[23,24,25]$ and explained theoretically in [26].

In the present study, we have determined the vesicle shapes and multidomain patterns by energy minimization. Thus, our calculations do not include the effects of thermally excited shape fluctuations. It will be interesting to study the impact of such fluctuations on the stability of the different morphologies and domain patterns. Likewise, it will be interesting to include the effect of spontaneous curvatures, which should increase the tendency for the formation of multi-domain patterns with more than two domains.

\section{References}

[1] R. Lipowsky, J. Phys. II France 2, 1825 (1992).

[2] F. Jülicher and R. Lipowsky, Phys. Rev. Lett. 70, 2964 (1993).

[3] C. Dietrich et al, Biophys. J. 80, 1417 (2001).

[4] T. Baumgart, S.T. Hess, and W.W. Webb, Nature 425, 821 (2003).

[5] S.L. Veatch and S.L. Keller, Biophys. J. 85, 3074 (2003)

[6] T. Baumgart, S. Das, W.W. Webb, and J.T. Jenkins, Biophys. J. 89, 1067 (2005). 
[7] S.L. Veatch and S.L. Keller, Phys. Rev. Lett. 94, 148101 (2005)

[8] K. Bacia, P. Schwille, and T. Kurzchalia, Proc. Natl. Acad. Sci. USA 102, $3272(2005)$.

[9] R. Dimova et al, Soft Matter 3, 817 (2007)

[10] S. Semrau, T. Idema, L. Holtzer, T. Schmidt, and C. Storm, Phys. Rev. Lett. 100, 088101 (2008)

[11] K. Simons and E. Ikonnen, Nature 389, 569 (1997).

[12] S. Mukherjee and F.R. Maxfield. Annu. Rev. Cell Dev. Biol. 20, 839 (2004).

[13] F. Jülicher and R. Lipowsky, Phys. Rev. E 53, 2670 (1996).

[14] S. Kumar, G. Gompper, and R. Lipowsky. Phys. Rev. Lett. 86, 3911 (2001).

[15] M. Laradji and P.B. Sunil Kumar, Phys. Rev. Lett. 93, 198105 (2004).

[16] G. Ayton, J.L. McWhirter, P. McMurtry, and G.A. Voth, Biophys. J. 88, 3855 (2005).

[17] R. Lipowsky and R. Dimova, J. Phys. Cond. Mat. 15, S31-S45 (2003).

[18] R. Lipowsky et al, J. Phys.: Condens. Matter 17, S2885 (2005).

[19] M. Yanagisawa, M. Imai, T. Masui, S. Komura, and T. Ohta, Biophys. J. 92, 115 (2007).

[20] W.T. Góźdź and G. Gompper, Phys. Rev. Lett. 80, 4213 (1998).

[21] H. Möhwald, in Structure and Dynamics of Membranes, volume 1 of Handbook of Biological Physics, pages 161-212 (Elsevier, Amsterdam, 1995).

[22] H. M. McConnell, and R. De Koker Langmuir 12, 4897 (1996)

[23] Tae-Young Yoon et al, Nature Matherials 5, 281 (2006).

[24] R. Parthasarathy, Ch. Yu, and J. T. Groves, Langmuir 22, 5095 (2006).

[25] R. Parthasarathy and J. T. Groves, Soft Matter 3, 24 (2007).

[26] B. Rozycki, T.R. Weikl and R. Lipowsky, Phys. Rev. Lett. 100, 098103 (2008).

[27] W. Helfrich, Z. Naturforsch. 28c, 693 (1973).

[28] M.D. Mitov. Compte rendues de l'Academie bulgare des Sciences 31, 513 (1978).

[29] U. Seifert and R. Lipowsky, in Structure and Dynamics of Membranes, volume 1 of Handbook of Biological Physics, pages 403-463 (Elsevier, Amsterdam, 1995). 
[30] W. Helfrich and W. Harbich, in Physics of Amphiphilic Layers, edited by J. Meunier, D. Langevin, and N. Boccara (Springer-Verlag, Berlin, 1987).

[31] R.H. Templer, B.J. Khoo, and J.M. Seddon, Langmuir 14, 7427 (1998).

[32] D. Benvegnu and H. McConnell, J. Phys. Chem. 96, 6820 (1992).

[33] M. Gandhavadi, D. Allende, A. Vidal, S. A. Simon, and T. J. McIntosh, Biophys. J. 82, 1469 (2002)

[34] C. Gebhardt, H. Gruler, and E. Sackmann, Z. Naturforsch. 32c, 581 (1977).

[35] P. L. Hansen, L. Miao, and J. H. Ipsen, Phys. Rev. E 58, 2311 (1998).

[36] S. Kumar, G. Gompper, and R. Lipowsky. Phys. Rev. E 60, 4610 (1999).

[37] J. L. Harden, F. C. MacKintosh, and P. D. Olmsted, Phys. Rev. E 72, 011903 (2005).

[38] M. Laradji and S. Kumar, Phys. Rev. E 73, 040901 (2006).

[39] A. J. Wagner, S. Loew, and S. May, Biophys. J. 93, 4268 (2007).

[40] K. Brakke, Exp. Math. 1, 141 (1992).

[41] M.P. do Carmo. Differential geometry of curves and surfaces. (PrenticeHall, Englewood Cliffs, 1976). 